\title{
Artéria umbilical única - Relato de caso
}

\section{Single umbilical artery - Case report}

Cíntia Lobo Sabino ${ }^{1}$, Isabella de Almeida Klein ${ }^{1}$, Juliana Ribeiro Fernandes ${ }^{1}$, Pedro Mateus da Silva Barreto ${ }^{1}$, Rebeca de Araujo Medeiros $^{1}$, Victor Fraga Ceotto ${ }^{1}$, Nayra Rodrigues Mazolli ${ }^{1}$, Maira Torres Silva ${ }^{1}$ e Adauto Dutra Moraes Barbosa ${ }^{1}$

'Universidade Federal Fluminense

\section{Palavras-chave:}

Artéria umbilical única; Anormalidades congênitas; Relações materno-fetais.

\section{Resumo}

Introdução: apresentamos um caso de artéria umbilical isolada, que, embora seja um achado visto frequentemente ao exame ultrassonográfico pré-natal e nas salas de parto, pode estar associado a outras malformações congênitas. Objetivo: relatar um caso de artéria umbilical única. Descrição do caso: apresentamos um caso de artéria umbilical isolada em um recém-nascido a termo, sem repercussões maternas ou fetais detectáveis. Discussão: a presença de artéria umbilical única pode estar associada a outras malformações congênitas. Na presença de fatores de risco, tais como idade avançada, alterações genéticas, diabetes mellitus durante a gravidez, história familiar de doença cardíaca congênita ou inseminação artificial, deverá ser realizado aconselhamento genético, ecocardiografia e ultrassonografia abdominal e geniturinária.

\section{Keywords:}

Single umbilical artery; Congenital abnormalities; Maternal-fetal relations.

\begin{abstract}
Introduction: we present a case of isolated umbilical artery that, although it is a frequently seen finding in the prenatal ultrasound examination and in the delivery rooms, may be associated with other congenital malformations. Objective: to report a case of single umbilical artery. Case description: we present a case of isolated umbilical artery in a full-term neonate with no detectable maternal or fetal repercussions. Discussion: the presence of a single umbilical artery may be associated with other congenital malformations. In the presence of risk factors such as advanced age, genetic alterations, diabetes mellitus during pregnancy, family history of congenital heart disease or artificial insemination, genetic counseling, echocardiography, and abdominal and genitourinary ultrasound should be performed.
\end{abstract}




\section{INTRODUÇÃO}

Na sua anatomia normal, o cordão umbilical possui três vasos: uma veia e duas artérias (Figura 1). As artérias conduzem o sangue não oxigenado do feto para a placenta e a veia transporta o sangue oxigenado na placenta para a circulação fetal.

A artéria umbilical única (AUU) é uma condição anormal do cordão umbilical em que uma artéria está ausente por agenesia primária ou atrofia secundária. A AUU é um achado comum, ${ }^{1}$ mas sua importância vem da associação com malformações ou síndromes. ${ }^{2}$

\section{DESCRIÇÃO DO CASO}

I. L. M., 31 anos de idade, 39 semanas e 5/7 dias de idade gestacional. Multípara, chegou à emergência obstétrica do Hospital Universitário Antônio Pedro (HUAP) com queixa de dor abdominal e perda de líquido.

Realizou cinco consultas pré-natais, sendo imunizada com as vacinas dT+dTpa e Influenza. A ultrassonografia do segundo trimestre mostrou a presença de uma única artéria umbilical. A ecocardiografia fetal, realizada no terceiro trimestre, não mostrou alterações. Testes complementares realizados no terceiro trimestre - entre eles cultura de urina, swab para GBS e sorologia materna para sífilis, Aids, hepatite e TORCH - foram negativos.

O parto foi vaginal, com apresentação cefálica com uma hora de bolsa rota e sem complicações. VDRL e anti-HIV na admissão também foram negativos. O exame de placenta mostrou dois vasos, uma artéria e uma veia, sem outras anormalidades.

Recém-nascida do sexo feminino, pesando $3.690 \mathrm{~g}$ (adequado para idade gestacional - AIG), com $52 \mathrm{~cm}$ de comprimento, $34 \mathrm{~cm}$ de perímetro cefálico, $33 \mathrm{~cm}$ de perímetro torácico e $31 \mathrm{~cm}$ de perímetro abdominal. Escore de Apgar nos $1^{\circ}$ e $5^{\circ}$ minutos foi de 8 e 9 , respectivamente.

O cordão umbilical examinado na sala de parto revelou uma artéria e uma veia (Figura 2), de acordo com o exame ultrassonográfico realizado durante a gravidez. Ela eliminou mecônio e urinou nas primeiras horas de vida, sem anormalidades.

Ao exame físico, realizado com seis horas de vida, a recém-nascida apresentava FC de 110 bpm, FR de 41 irpm e boas condições gerais; era ativa e reativa ao estímulo manual; normocorada e hidratada; apresentava acrocianose discreta e anictérica. Reflexos primitivos presentes. A fontanela anterior media $2 \mathrm{~cm} \chi 1,5 \mathrm{~cm}$ e a posterior encontrava-se fechada. Exame cardiopulmonar com ausculta normal. Inspeção e palpação do abdome eram normais, sem massas ou visceromegalias.
Figura 1 - Cordão umbilical normal com três vasos, duas artérias e uma veia

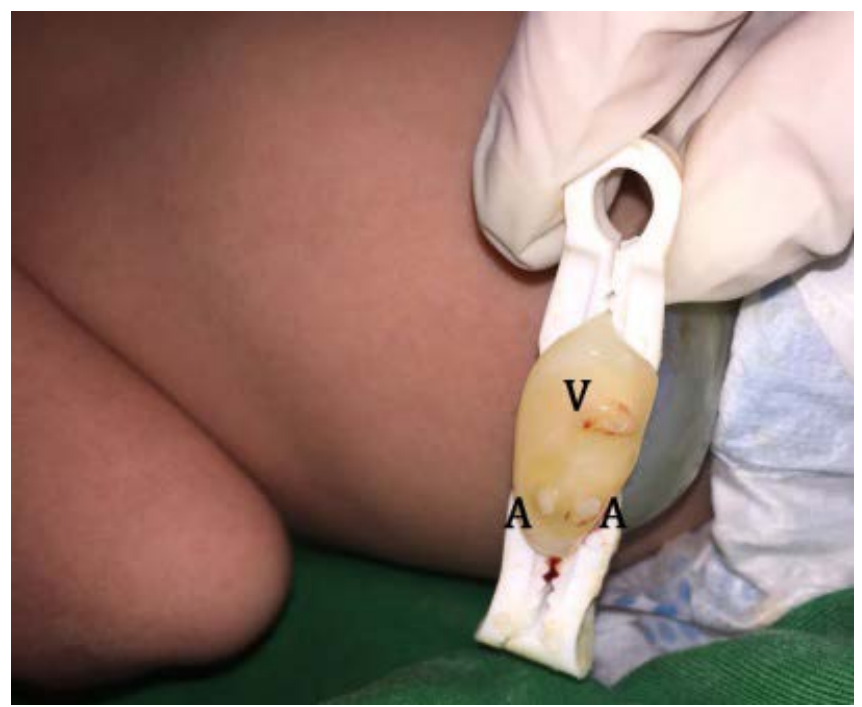

Fonte: Arquivo pessoal (2018).

Figura 2 - Cordão umbilical com dois vasos, uma artéria e uma veia

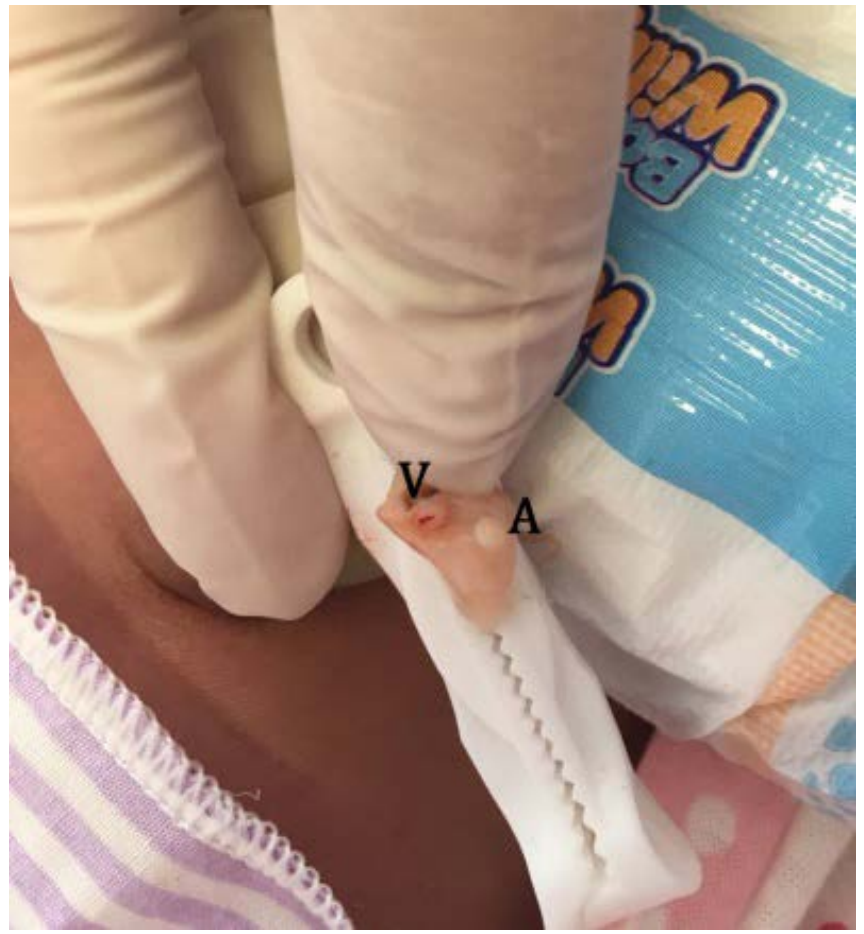

Fonte: Arquivo pessoal (2018).

Genitália feminina normal, gênero compatível. Testes de Barlow e de Ortolani negativos.

Ainda na maternidade, foram realizados testes do reflexo vermelho, teste de oximetria e de avaliação de emissão otoacústica, todos normais. Finalmente, foram realizados ultrassonografia do abdome e do trato urinário, que não apresentaram alterações. 
Tanto a mãe quanto a recém-nascida receberam alta hospitalar, com diretrizes gerais.

\section{DISCUSSÃO}

Os mecanismos etiopatogênicos da formação da AUU ainda permanecem incertos, contudo algumas teorias propostas, tais como a agenesia primária de uma artéria umbilical, na qual não há diferenciação em duas artérias, durante sua gênese, e a ocorrência de um processo secundário de atresia, a partir de uma das artérias umbilicais já normalmente formadas, são as mais conhecidas. ${ }^{3}$

Algumas desordens vasculares do cordão umbilical podem estar associadas à AUU e didaticamente podem ser enquadradas em quatro tipos: ${ }^{4}$

a) tipo I - cordão umbilical possui dois vasos, sendo uma artéria umbilical de origem alantoide, que se conecta com a artéria ilíaca, e uma veia umbilical esquerda;

b) tipo II - cordão umbilical possui dois vasos, sendo uma artéria umbilical de origem vitelina, que se comunica com a artéria mesentérica superior, e uma veia umbilical esquerda;

c) tipo III - cordão umbilical possui três vasos, sendo uma artéria umbilical (origem alantoide ou vitelina) e duas veias umbilicais, uma esquerda e uma direita;

d) tipo IV - cordão umbilical possui dois vasos, sendo uma artéria umbilical (origem alantoide ou vitelina) e uma veia umbilical direita.

Quase a totalidade dos casos se enquadra no primeiro tipo, inclusive o encontrado neste trabalho.

A AUU tem uma incidência de 0,1-1,6\%, dependendo da população estudada, ${ }^{1,2}$ sendo ainda mais frequente em partos múltiplos e abortos. ${ }^{5}$ Sua importância vem da possível associação com anormalidades cromossômicas e malformações do trato geniturinário, ${ }^{1,5}$ gastrointestinal, ${ }^{6}$ cardiovascular ${ }^{7}$ e do sistema musculoesquelético. ${ }^{1}$

A AUU é vista como parte de uma síndrome em 36,4\% dos casos, dentre os quais $10,9 \%$ possuem anormalidade cromossômica. ${ }^{2}$ Além disso, o diagnóstico de AUU também está fortemente associado a desfechos desfavoráveis no período perinatal, ${ }^{8}$ tais como acidose ( $\mathrm{pH}$ diminuído no sangue do cordão), ${ }^{9}$ escore de Apgar baixo no primeiro minuto, ${ }^{10}$ prematuridade, baixo peso ao nascer, ${ }^{10}$ maior incidência de internação em unidade de terapia intensiva e maior taxa de cesariana. ${ }^{9}$

Embora o baixo peso ao nascer seja muito frequente em recém-nascidos com AUU, sua fisiopatologia ainda é desconhecida ${ }^{9}$ e a presença de anormalidades placentárias seria uma possível explicação. Neste âmbito, observou-se que, nos casos de artéria umbilical isolada, há uma maior incidência de má perfusão fetal de alto grau e uma menor incidência de perfusão materna, ${ }^{11}$ não havendo diferença nos resultados quanto ao lado da artéria ausente. ${ }^{12}$ No entanto, nenhuma associação com outras patologias placentárias foi observada ${ }^{11}$ no caso descrito.

Com relação ao seu diagnóstico, a AUU pode ser observada à ultrassonografia do segundo trimestre de gestação, embora possa ser estabelecido com boa precisão mesmo às 12 semanas de gestação. ${ }^{13}$

Chen K. e colaboradores ${ }^{14}$ observaram em sua coorte que não houve aumento de risco para cardiopatia congênita (CC) em gestações sem fatores de risco. Porém, na presença de um ou mais fatores de risco, a incidência de CC era de 15,6\%, sendo observados coarctação da aorta, defeito de septo ventricular e defeito de septo atrioventricular entre as mais frequentes. Assim, os autores sugerem que a triagem com ecocardiografia em fetos com AUU não precisa ser universal e pode ser restrita a gravidezes com fatores de risco, ${ }^{14}$ fato corroborado por Gurram et al. ${ }^{15}$

Assim, alertamos o pediatra que, embora a ocorrência de artéria umbilical isolada usualmente não cause grande repercussão ao feto, quando associada a quaisquer fatores de risco, estará indicado aconselhamento genético, ecocardiografia e avaliação ultrassonográfica abdominal e de aparelho geniturinário. 


\section{REFERÊNCIAS}

1 Murphy-Kaulbeck L, Dodds L, Joseph KS, Van den Hof M. Single umbilical artery risk factors and pregnancy outcomes. Obstet Gynecol. 2010;116(4):843-50.

2 Austin-Ward ED, Nazer JH. Arteria umbilical unica y malformaciones asociadas: experiencia en el Hospital Clinico de la Universidad de Chile. Rev. Chil. Pediatr. 1998;69(5):195-9.

3 Persutte WH, Hobbins J. Single umbilical artery: a clinical enigma in modern prenatal diagnosis. Ultrasound Obstet Gynecol. 1995;6(3):216-29.

4 Blackburn, W, Cooley NR. The umbilical cord. In: Stevenson RE, Hall JG, Goodman RM (Eds.). Human malformations and related anomalies. Vol. II. New York: Oxford University Press; 1993. p. 1081-141.

5 Nayak SS, Shukla A, Girisha KM. Anomalies associated with single umbilical artery at perinatal autopsy. Indian Pediatr. 2015;52(1):73-4

6 McMurran AE, McMahon SV, Walker GM. Intestinal associations of a single umbilical artery. Arch Dis Child Fetal Neonatal Ed. 2015;100(3):F263

7 Araujo Júnior E, Palma-Dias R, Martins WP, Reidy $\mathrm{K}$, da Silva Costa F. Congenital heart disease and adverse perinatal outcome in fetuses with confirmed isolated single functioning umbilical artery. J Obstet Gynaecol. 2015;35(1):85-7.

8 Horton AL, Barroilhet L, Wolfe HM. Perinatal outcomes in isolated single umbilical artery. Am J Perinatol.
2010;27(4):321-4.

9 Naveiro-Fuentes M, Carrillo-Badillo MP, Malde-Conde J, Gallo-Vallejo JL, Puertas-Prieto A. Perinatal outcomes in singleton pregnancies with a single umbilical artery. J Matern Fetal Neonatal Med. 2016;29(10):1562-5.

10 Luo X, Zhai S, Shi N, Li M, Cui S, Xu Y et al. The risk factors and neonatal outcomes of isolated single umbilical artery in singleton pregnancy: a meta-analysis. Scientific Reports. 2017;7:7396.

11 Battarbee AN, Palatnik A, Ernst LM, Grobman WA. Placental abnormalities associated with isolated single umbilical artery in small-for-gestational-age births. Placenta. 2017;59:9-12.

12 Wu YP, Tsai HF, Cheng YC, Kang L, Tsai PY, Yu CH et al. Prenatal sonographic diagnosis of single umbilical artery: emphasis on the absent side and its relation to associated anomalies. Taiwan J Obstet Gynecol. 2014;53(2):197-201.

13 Martínez-Payo C, Cabezas E, Nieto Y, Ruiz de Azúa M, García-Benasach F et al. Detection of single umbilical artery in the first trimester ultrasound: its value as a marker of fetal malformation. BioMed Res Intern Art. 2014. DOI: http://dx.doi.org/10.1155/2014/548729

14 Chen K, Akoma U, Anderson A, Mertz H, Quartermain MD. Prenatally diagnosed single umbilical artery: the role and relationship of additional risk factors in the fetus for congenital heart disease. J Clin Ultrasound. 2016;44(2):113-7.

15 Gurram P, Figueroa R, Sipusic E, Kuhnly N, Clark $\mathrm{S}$, Janicki MB. Isolated single umbilical artery and fetal echocardiography: a 25 -year experience at a tertiary care city hospital. J Ultrasound Med. 2018;37(2):463-68. 